\title{
Risk factors for rapid progressive neurological deterioration in patients with cervical spondylotic myelopathy
}

\author{
Weiyang Zhong, Lin Wang, Tianji Huang and Xiaoji Luo*
}

\begin{abstract}
Background: The rapid progressive cervical spondylotic myelopathy (rp-CSM) which had a course of CSM less than 1 month and suffered rapidly progressive neurological deterioration had few reports. Therefore, it is important for us to recognize the pathophysiology of CSM especially the rp-CSM. The study aimed to investigate the risk factors for rapidly progressive (rp) neurological deterioration in patients with cervical spondylotic myelopathy (CSM).

Methods: A total of 159 patients were reviewed and divided into an rp-CSM group and a chronic-CSM (c-CSM) group. Various clinical indexes, including age, sex, Japanese Orthopaedic Association (JOA) score, intramedullary MR T2-hyperintensity, congenital/degenerative spinal stenosis, and local type of ossification of the posterior longitudinal ligament (OPLL), were analyzed, and independent risk factors were investigated.

Results: Thirty-four of 159 patients (21.4\%) were diagnosed with rp-CSM. All patients were followed up for a mean of $68.56 \pm 14.00$ months in the rp-CSM group and $62.66 \pm 19.95$ months in the c-CSM group. No significant difference was found in sex, mean age, smoking and drinking status, diabetes mellitus (DM), hypertension, surgery time, blood loss, JOA score, degenerative spinal stenosis, or OPLL (local). Univariate analyses demonstrated that rpCSM patients tended to have MR T2-hyperintensity, longer hospital stay, shorter waiting time for surgery, more congenital spinal stenosis, and worse neurological function and to prefer more posterior surgeries than c-CSM patients. A multiple logistic regression analysis showed that congenital spinal stenosis and MR T2-hyperintensity were independently related to the presence of rp-CSM.
\end{abstract}

Conclusions: MR T2-hyperintensity and congenital spinal stenosis were risk factors for rp-CSM. Although neurological function deteriorates rapidly, early surgical decompression is recommended and can achieve good neurological recovery after surgery, indicating that rp-CSM could be a reversible condition.

Keywords: Cervical spondylotic myelopathy "Risk factor "MR T2-hyperintensity

\section{Background}

Cervical spondylotic myelopathy (CSM) is usually considered a slow, progressive spinal disease that is a major cause of spinal cord dysfunction, particularly in elderly individuals, but it is often delayed to diagnosis. Not all patients with presence of spinal cord compression have

* Correspondence: cy2982@163.com

Department of Orthopedic Surgery, The First Affiliated Hospital of

Chongqing Medical University, Chongqing 400016, P. R. China symptoms, and the progression ofCSM varies by patients. Magnetic resonance imaging (MRI) is an important imaging choice for patients with suspected CSM and the patients suspected of CSM is recommended to spine surgeon immediately and the severe patients can be typically treated surgically [1-3].

However, we focused on chronic year-by-year neurological exacerbation of CSM. A few patients with CSM suffered rapidly progressive neurological deterioration,

(c) The Author(s). 2021 Open Access This article is licensed under a Creative Commons Attribution 4.0 International License, which permits use, sharing, adaptation, distribution and reproduction in any medium or format, as long as you give appropriate credit to the original author(s) and the source, provide a link to the Creative Commons licence, and indicate if changes were made. The images or other third party material in this article are included in the article's Creative Commons licence, unless indicated otherwise in a credit line to the material. If material is not included in the article's Creative Commons licence and your intended use is not permitted by statutory regulation or exceeds the permitted use, you will need to obtain permission directly from the copyright holder. To view a copy of this licence, visit http://creativecommons.org/licenses/by/4.0/ The Creative Commons Public Domain Dedication waiver (http://creativecommons.org/publicdomain/zero/1.0/) applies to the data made available in this article, unless otherwise stated in a credit line to the data. 
particularly with walking disorders. Compared with chronic CSM (c-CSM), there have been few reports on the characteristics of rapidly progressive CSM (rp-CSM) $[4,5]$. Therefore, it is important for us to recognize rp-CSM and the pathogenesis of rp-CSM. This study investigated the characteristics and potential risk factors for rp-CSM.

\section{Material and methods Population selection}

This study was approved by the Institutional Review Board of our hospital and was conducted according to the principles of the Declaration of Helsinki. All the patients provided their written informed consent to participate in our study prior to the storage of their data in the hospital database. The cases of patients visiting our hospital between January 2010 and January 2014 were retrospectively reviewed. rp-CSP was defined as when the patients had a course of CSM less than 1 month and suffered rapidly progressive neurological deterioration, causing difficulty in maintaining a standing posture or walking without support $[4,5]$. c-CSM was defined as when the patients had a course of CSM of more than 1 month $[4,5]$. Inclusion criteria: severe patients who failed conservative treatment, suffered rp-CSM or c-CSM, and underwent surgery 3-4 days after hospitalization. Exclusion criteria include metastatic fractures, primary tumors, a history of any cervical spine surgery, high-energy trauma fractures, a history of cervical trauma, or infections.

\section{Surgical technique}

All surgeries were performed by the same senior spinal surgeon. Anterior surgeries included anterior cervical discectomy and fusion (ACDF), anterior cervical corpectomy and fusion (ACCF), and hybrid procedures. Posterior surgeries included open-door laminoplasty or laminectomy and fusion. Postoperatively, all patients were required to wear a Philadelphia hard cervical collar for 6 to 8 weeks. In addition, the patients began step-bystep training of their neck muscles.

\section{Outcome assessment}

The neurological status was evaluated preoperatively and at the final follow-up (FU) based on the Japanese Orthopaedic Association (JOA) score. The affected level of intramedullary hyperintensity lesions was assessed on a magnetic resonance imaging (MRI) T2-weighted system (Siemens, Germany). Congenital spine stenosis was assessed by the Torg-Pavlov ratio, which was defined as follows: on the X-ray, the ratio of the sagittal diameter of the spinal canal to the sagittal diameter of the vertebral body was less than 0.75 , while the Palvov value of degenerative spine stenosis was more than 0.75 and less than 1.0 .

\section{Statistics}

The statistical analysis was performed using univariate analyses to compare the preoperative and postoperative outcomes of the two groups, and a multivariate logistic regression analysis was performed to determine the factors associated with the presence of rp-CSM using the Statistic Analysis System (SAS Institute Inc., Cary, NC, USA). The results are expressed as the group means \pm SD. Differences with a $P$ value $<0.05$ were considered significant.

\section{Results}

Thirty-four of 159 patients (21.4\%) were diagnosed with rp-CSM. All patients were followed up for a mean of $68.56 \pm 14.00$ months in the rp-CSM group and $62.66 \pm$ 19.95 months in the c-CSM group $(P>0.05)$. No significant difference was found in sex, mean age, smoking and drinking status, diabetes mellitus (DM), hypertension, surgery time, blood loss, JOA score, congenital/degenerative spinal stenosis, or OPLL (local) $(P>0.05)$ between the groups. A comparison of the surgical approaches showed that there was no significant difference between anterior surgery and anterior combined with posterior surgery except the posterior approach (Table 1).

Univariate analyses demonstrated that rp-CSM patients tended to have MR T2-hyperintensity (rp-CSM $88.2 \%$ vs. c-CSM $24.8 \% ; P<0.0001$ ), longer hospital stay (rp-CSM $15.97 \pm 6.92$ days vs. c-CSM $12.78 \pm 4.67$ days; $P=0.0019$ ), shorter waiting time for surgery (rp-CSM $0.94 \pm 0.43 \mathrm{~m}$ vs. c-CSM $113.3 \pm 108.8 \mathrm{~m} ; P<0.0001)$, more congenital spinal stenosis (rp-CSM $48.8 \%$ vs. cCSM 10.4\%; $P=0.0181$ ), and worse neurological function (JOA: rp-CSM $8.73 \pm 1.73$ vs. c-CSM $13.94 \pm 1.82$; $P<0.0001)$ and to prefer more posterior surgeries (rpCSM $20.6 \%$ vs. c-CSM 8\%; $P=0.0188$ ) (Table 1 ).

A multiple logistic regression analysis showed that congenital spinal stenosis (odds ratio $(\mathrm{OR})=3.95,95 \%$ confidence interval (CI) 1.30-17.05, $P=0.030$ ) and MR T2-hyperintensity $(\mathrm{OR}=12.3,95 \%$ CI $1.49-78.94, P=$ 0.021 ) were independently related to the presence of rpCSM (Fig. 1).

\section{Discussion}

With the increasing incidence of CSM, the clinical presentation and natural history of CSM vary, and CSM carries a high risk for disability [1-3, 6-10]. The aim of MRI is to help diagnose and manage patients earlier, especially those with rp-CSM [11]. In our study, $88.2 \%$ of rp-CSM patients presented with MR T2-hyperintense lesions, which was a risk factor, as reported previously by Morishita et al. [4, 5, 10-13]. On MRI, T2-hyperintense changes are considered to reflect the pathologies of spinal cord injury, such as edema, gliosis, demyelination, 
Table 1 Clinical information between groups

\begin{tabular}{llll}
\hline & rp-CSM & c-CSM & P value \\
\hline No. of patients $(n)$ & 34 & 125 & \\
Males/females ( $n$ ) & $19 / 15$ & $68 / 57$ & 0.8786 \\
Mean age & $57.24 \pm 11.86$ & $56.51 \pm 11.64$ & 0.7494 \\
Smoker(\%) & $32.4 \%$ & $16.8 \%$ & 0.1286 \\
Drink (\%) & $23.5 \%$ & $17.6 \%$ & 0.6589 \\
Diabetes mellitus (\%) & $11.8 \%$ & $10.4 \%$ & 0.8208 \\
Hypertension (\%) & $35.3 \%$ & $15.2 \%$ & 0.1020 \\
Hospital stay & $15.97 \pm 6.92$ & $12.78 \pm 4.67$ & 0.0019 \\
Waiting period to surgery (m) & $0.94 \pm 0.43$ & $25.80 \pm 35.24$ & $<0.0001$ \\
Blood loss (ml) & $160.0 \pm 214.0$ & $113.3 \pm 108.8$ & 0.0814 \\
Surgery time (min) & $158.4 \pm 58.39$ & $145.7 \pm 57.66$ & 0.2568 \\
MR T2-hyperintensity (\%) & $88.2 \%$ & $24.8 \%$ & $<0.0001$ \\
Congenital spinal stenosis (\%) & $8.8 \%$ & $10.4 \%$ & 0.7881 \\
Degenerative spinal stenosis (\%) & $70.6 \%$ & $56.8 \%$ & 0.1479 \\
OPLL (local) (\%) & $5.9 \%$ & $7.2 \%$ & 0.7900 \\
JOA & & & \\
$\quad$ Pre-operation & $14.00 \pm 1.97$ & $13.94 \pm 1.82$ & 0.8760 \\
Final FU & $16.62 \pm 0.78$ & $16.57 \pm 0.75$ & 0.7360 \\
Surgery approach & & & \\
$\quad$ Anterior surgery & $76.5 \%$ & $90.4 \%$ & 0.0689 \\
$\quad$ Posterior surgery & $20.6 \%$ & $8.8 \%$ & 0.0188 \\
$\quad$ Anterior + posterior surgery & $2.9 \%$ & $0.8 \%$ & 0.3236 \\
$\quad$ Mean FU (m) & $68.56 \pm 14.00$ & $62.66 \pm 19.95$ & 0.1076 \\
\hline
\end{tabular}

and myelomalacia. The high frequency of MR T2hyperintensity might be indicative of spinal edema followed by acute spinal ischemia. This finding suggested the existence of microvascular lesions, causing glucose dysfunction and insufficient blood supply in the cervical spinal cord, which ultimately resulted in acute spinal cord ischemia observed as MR T2-hyperintensity. Congenital spinal stenosis, as a risk factor, could reduce the volume of the spinal canal, which could aggravate spinal cord injury. More attention should be paid to CSM patients with congenital spinal stenosis who could suffer rapid progressive neurological deterioration, and strict follow-up is recommended.

It was reported that there is a relationship between diabetes and a poor outcome in patients with CSM and that microvascular disorder caused by DM pathophysiology could affect or aggravate CSM [14, 15]. However, in our study, DM, smoking status, and hypertension were not related to rp-CSM. We agree with the coexistence of comorbidities that could be a potential risk of microvascular dysfunction and could not fully explain and understand the pathophysiological condition in patients with CSM, especially rp-CSM. More basic experiments are needed to investigate the pathogenesis of rpCSM.

In the patients with rp-CSM and c-CSM, there was a significant difference in preoperative JOA score, and the neurological function of rp-CSM was much worse than that of c-CSM. The JOA score at the final FU was significantly improved, but there was no significant difference between the groups. The surgical treatments were both effective for rp-CSM and c-CSM patients. Furthermore, the rp-CSM patients showed good neurological recovery after surgery, indicating that this condition could be reversible. The optimal surgical timing and approach are still controversial and can vary depending on multiple factors, such as the location of the spinal cord compression, affected levels, and patient comorbidities [12, 13, 15-19]. In our study, rp-CSM patients had a shorter waiting period to surgery because of rapidly progressive neurological deterioration, and earlier decompression was considered. According to the direction of spinal cord compression and the number of surgical segments, different surgical approaches were preferred. In our study, in both groups, more patients were performed by the anterior approach without significant difference.

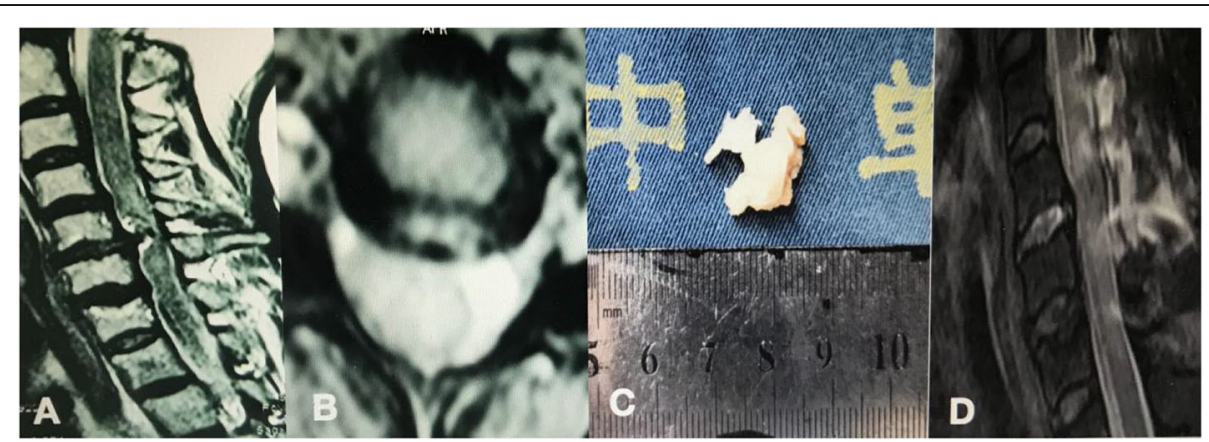

Fig. 1 A 66-year-old female patient presented with Brown-Sequard syndrome of the left limbs and trunk for 3 days and was diagnosed with rpCSM. a, b MRI showed spinal compression and T2-hyperintensity at the C5/6 disk level with edema at the C4-5 and C5-6 levels, probably due to spinal cord ischemia. c The patient underwent laminectomy and fusion, and the large disk was removed. $\mathbf{d}$ Postoperative MRI showed that decompression was satisfactory and neurological symptoms improved significantly 
The rp-CSM patients had spinal stenosis performed by posterior surgeries, which had longer hospital stays, while more anterior surgeries were performed inc-CSM patients. Regardless of the surgical approach, early decompression could achieve good neurological recovery without missing the window of opportunity for surgery.

However, our study had a few limitations. First, the retrospective nature of the small-sample study may be associated with bias. Second, the definition of rp-CSM was clinically discussed, but more clues regarding biomechanics, cytology, and pathology are needed. The timing of surgery for rp-CSM patients was difficult to determine, but it mostly depended on the neurological status and on the surgeon's management or experience. Third, our study investigated whether MR T2hyperintensity was associated with rp-CSM. T1weighted, T2-weighted, or STIR sequences of the MR signal changes will be studied to gain more understanding of rp-CSM. In the future, prospective, randomized, grouped studies with long-term follow-up periods are needed.

\section{Conclusion}

In our study, MR T2-hyperintensity and congenital spinal stenosis were risk factors for rp-CSM. Although the neurological impairment deteriorated rapidly, early surgical decompression is recommended and could achieve good neurological recovery after surgery, indicating a reversible condition.

\section{Abbreviations \\ CSM: Cervical spondylotic myelopathy; rp-CSM: Rapid progressive cervical spondylotic myelopathy; C-CSM: Chronic-CSM; JOA: Japanese Orthopaedic Association; OPLL: Ossification of the posterior longitudinal ligament; ACDF: Anterior cervical discectomy and fusion; ACCF: Anterior cervical corpectomy and fusion; DM: Diabetes mellitus}

\section{Acknowledgements}

Not applicable.

\section{Authors' contributions}

WYZ and XJL designed the study. LW and TJH collected the data. WYZ, XJL, $L W$, and TJH performed the statistical analysis and drafted and revised the manuscript. WYZ revised the manuscript. All authors read and approved the final manuscript

\section{Funding}

The study was supported by the hospital training funding (PYJJ2019-08) and the study was supported by the medical senior talents programme of Chongqing (2019GDRC001).

\section{Availability of data and materials}

The datasets generated and/or analyzed during the current study are not publicly available due to the data is confidential patient data but are available from the corresponding author on reasonable request.

\section{Ethics approval and consent to participate}

The Institutional Review Board of the First Affiliated Hospital of Chongqing Medical University approved this study and conducted in accordance with the Declaration of Helsinki. All participants provided written informed consent before their data were stored in our hospital database and used for study purposes.
Consent for publication

Not applicable.

\section{Competing interests}

The authors declare that they have no competing interests.

Received: 24 October 2020 Accepted: 11 January 2021

Published online: 21 January 2021

\section{References}

1. Singh A, Tetreault L, Casey A, Laing R, Statham P, Fehlings MG. A summary of assessment tools for patients suffering from cervical spondylotic myelopathy: a systematic review on validity, reliability and responsiveness. Eur Spine J. 2015;24(suppl 2):209-28.

2. Wu JC, Ko CC, Yen YS, et al. Epidemiology of cervical spondylotic myelopathy and its risk of causing spinal cord injury: a national cohort study. Neurosurg Focus. 2013;35:E10.

3. Sadasivan KK, Reddy RP, Albright JA. The natural history of cervical spondylotic myelopathy. Yale J Biol Med. 1993;66:235-42.

4. Takasawa E, Sorimachi Y, lizuka Y, et al. Risk factors for rapidly progressive neurological deterioration in cervical spondylotic myelopathy. Spine (Phila Pa 1976). 2019:44(12):E723-30.

5. Morishita Y, Matsushita A, Maeda T, et al. Rapid progressive clinical deterioration of cervical spondylotic myelopathy. Spinal Cord. 2015;53(5): 408-12.

6. Cooper PR. Cervical spondylotic myelopathy. Contemp Neuro-Surg. 1997; 19(25):1-7.

7. Ghogawala Z, Benzel EC, Heary RF, et al. Cervical spondylotic myelopathy surgical trial: randomized, controlled trial design and rationale. Neurosurgery. 2014;75:334-46.

8. Ohya J, Chikuda H, Oichi T, et al. Perioperative stroke in patients undergoing elective spinal surgery: a retrospective analysis using the Japanese diagnosis procedure combination database. BMC Musculoskelet Disord. 2015;16:276.

9. Fehlings MG, Tator $\mathrm{CH}$, Linden RD. The effect of nimodipine and dextran on axonal function and blood flow following experimental spinal cord injury. J Neurosurg. 1989;71:403-16.

10. Shields $C B$, Zhang YP, Shields $L B$, et al. The therapeutic window for spinal cord decompression in a rat spinal cord injury model. J Neurosurg Spine. 2005;3:302-7.

11. Nouri A, Tetreault L, Zamorano JJ, et al. The relationship between MRI signal intensity changes, clinical presentation, and surgical outcome in degenerative cervical myelopathy: analysis of a global cohort. Spine (Phila Pa 1976). 2015;40:171-8.

12. Fan X, Wang Z, Gao X, et al. The change of cervical sagittal parameters plays an important role in clinical outcomes of cervical spondylotic myelopathy after multi-level anterior cervical discectomy and fusion. $J$ Orthop Surg Res. 2019;14:429.

13. Xu Y, Chen F, Wang Y, et al. Surgical approaches and outcomes for cervical myelopathy with increased signal intensity on T2-weighted MRl: a metaanalysis. J Orthop Surg Res. 2019:14:224.

14. Dokai T, Nagashima H, Nanjo Y, et al. Surgical outcomes and prognostic factors of cervical spondylotic myelopathy in diabetic patients. Arch Orthop Trauma Surg. 2012;132:577-82.

15. Luo J, Cao K, Huang S, et al. Comparison of anterior approach versus posterior approach for the treatment of multilevel cervical spondylotic myelopathy. Eur Spine J. 2015;24:1621-30.

16. Zhu B, Xu Y, Liu X, Liu Z, Dang G. Anterior approach versus posterior approach for the treatment of multilevel cervical spondylotic myelopathy: a systemic review and meta-analysis. Eur Spine J. 2013;22:1583-93.

17. MCAllister BD, Rebholz BJ, Wang JC. Is posterior fusion necessary with laminectomy in the cervical spine? Surg Neurol Int. 2012:3(suppl 3):S225-31.

18. Lao $L$, Zhong $G$, Li X, et al. Laminoplasty versus laminectomy for multi-level cervical spondylotic myelopathy: a systematic review of the literature. J Orthop Surg Res. 2013;8:45

19. Cusick JF. Pathophysiology and treatment of cervical spondylotic myelopathy. Clin Neurosurg. 1991;37:661-81.

\section{Publisher's Note}

Springer Nature remains neutral with regard to jurisdictional claims in published maps and institutional affiliations. 